\title{
MiR-199a Inhibits Tumor Growth and Attenuates Chemoresistance by Targeting K-RAS via AKT and ERK Signalings
}

Wei $\mathrm{Li}^{1+}$, Lin Wang ${ }^{2+}$, Xiang-Bo $\mathrm{Ji}^{2}$, Li-Hong Wang ${ }^{2}$, Xin $\mathrm{Ge}^{3}$, Wei-Tao Liu ${ }^{3}$, Ling Chen ${ }^{1}$, Zhong Zheng ${ }^{1}$, Zhu-Mei Shi ${ }^{3}$, Ling-Zhi Liu ${ }^{4}$, Marie C. Lin ${ }^{2}$, Jie-Yu Chen ${ }^{1 *}$ and Bing-Hua Jiang ${ }^{2,3,4 *}$

${ }^{1}$ Department of Pathology, Affiliated Drum Tower Hospital of Nanjing University Medical School, Nanjing, China, ${ }^{2}$ Institute of Medical and Pharmaceutical Sciences, The Academy of Medical Sciences, Zhengzhou University, Zhengzhou, China, ${ }^{3}$ Key Laboratory of Human Functional Genomics of Jiangsu Province, Department of Pathology, Nanjing Medical University, Nanjing, China, ${ }^{4}$ Department of Pathology, Carver College of Medicine, The University of lowa, lowa, IA, United States

OPEN ACCESS

Edited by:

Pascale Cohen,

Université Claude Bernard

Lyon 1, France

Reviewed by:

Jessica Dal Col,

University of Salerno, Italy

Vishwa Jeet Amatya,

Hiroshima University, Japan

*Correspondence: Jie-Yu Chen

crucianfish@hotmail.com

Bing-Hua Jiang

binghiiang@yahoo.com

${ }^{\dagger}$ These authors have contributed equally to this work

Specialty section:

This article was submitted to Molecular and Cellular Oncology,

a section of the journal

Frontiers in Oncology

Received: 09 July 2019 Accepted: 30 September 2019

Published: 15 October 2019

Citation:

Li W, Wang L, Ji X-B, Wang L-H, Ge X, Liu W-T, Chen L, Zheng Z,

Shi Z-M, Liu L-Z, Lin MC, Chen J-Y

and Jiang B-H (2019) MiR-199a

Inhibits Tumor Growth and Attenuates

Chemoresistance by Targeting K-RAS

via AKT and ERK Signalings.

Front. Oncol. 9:1071.

doi: 10.3389/fonc.2019.01071
Glioma is the most malignant brain tumors in the world, the function and molecular mechanism of microRNA-199a (miR-199a) in glioma is not fully understood. Our research aims to investigate miR-199a/K-RAS axis in regulation of glioma tumor growth and chemoresistance. The function of miR-199a in glioma was investigated through in vitro and in vivo assays. We found that miR-199a in tumor tissues of glioma patients was significantly downregulated in this study. Kinase suppressor of ras 1 (K-RAS), was indicated as a direct target of miR-199a, as well as expression levels of K-RAS were inversely correlated with expression levels of miR-199a in human glioma specimens. Forced expression of miR-199a suppressed AKT and ERK activation, decreased HIF-1 $\alpha$ and VEGF expression, inhibited cell proliferation and cell migration, forced expression of K-RAS restored the inhibitory effect of miR-199a on cell proliferation and cell migration. Moreover, miR-199a renders tumor cells more sensitive to temozolomide (TMZ) via targeting K-RAS. In vivo experiment validated that miR-199a functioned as a tumor suppressor, inhibited tumor growth by targeting K-RAS and suppressed activation of AKT, ERK and HIF-1 $\alpha$ expression. Taken together, these findings indicated that miR-199a inhibits tumor growth and chemoresistance by regulating K-RAS, and the miR-199a/K-RAS axis is a potential therapeutic target for clinical intervention in glioma.

Keywords: miR-199a, glioma, K-RAS, chemoresistance, tumorigenesis

\section{INTRODUCTION}

Malignant gliomas, as most common brain tumors around the world $(1,2)$, are classified according to their degree of malignancy as Grades I to IV $(3,4)$. Glioma clinical treatment includes surgery, chemotherapy and radiotherapy $(5,6)$. The most malignant grade IV glioma, glioblastomamultiforme (GBM), has an average life expectancy of only 15 months (7). Investigation of glioma carcinogenesis mechanisms would improve clinical diagnosis, drug therapy and prevention of glioma.

MicroRNAs (miRNAs) are a class of endogenous 18-22 nucleotides RNA molecules $(8,9)$, which always bind to the $3^{\prime}$-untranslated region (UTR) of specific target mRNAs, and then regulate 
expression of several genes at the post-transcriptional level (10-14). Accumulated evidence has clearly demonstrated that aberrant miRNA expression profiles (15) and dysregulations of specific miRNAs and their target genes, are closely associated with tumor initiation and promotion in glioma $(16,17)$. In particular, the miR-199a has demonstrated to suppress tumor growth in a variety of cancers including esophageal, liver, and colorectal cancers. So far, the reported miR-199a downstream target genes include oncogenes PHLPP1, E2F3, FZD6/7, HK2, and MAP3K11 (18-23), which are functioned in pathogenesis of various cancers.

K-RAS, which is reported as a family member of Ras oncogene, has involved in regulation of some cellular signal transductions (24). K-RAS is implicated in the pathogenesis of various tumors, such as GBM and pilocytic astrocytoma $(25,26)$. Activation of K-RAS could promote the activations of several downstream molecules, such as MAPK and ERK to regulate biological processes $(27,28)$.

In this study for the first time in glioma, we demonstrated the loss of miR-199a, and that K-RAS is an important direct target gene of miR-199a. Results from in vitro studies in human glioma U87 and U251 cells indicated that forced expression of miR199a downregulated K-RAS signaling and suppressed cancer development and Temozolomide (TMZ) chemoresistance. The forced miR-199a overexpression also inhibited AKT and ERK1/2 pathways, through K-RAS signaling. The in vivo studies further demonstrated that over-express of miR-199a exhibited reduced tumor growth with down-regulated K-RAS/AKT/ERK/HIF$1 \alpha$ signalings. These results suggested that the loss of miR199a/K-RAS signaling in glioma plays a pivotal role in glioma progression, and it is a potential novel targets for future clinical treatment.

\section{MATERIALS AND METHODS}

\section{Specimen Collection}

Human glioma specimens $(n=24)$ and normal brain tissues $(n=9)$ were collected from patients in Nanjing University Medical School, China. Samples were obtained from patients with informed consents and were histologically classified by clinical pathologist.

\section{Cell Culture and Reagents}

Human glioma cells (U87, U251) were cultured in DMEM medium. Antibodies against anti-GAPDH and anti-HIF- $1 \alpha$ were purchased from Bioworld Technology (Atlanta, USA). Antibodies against antip-AKT (Ser473), anti-AKT, anti-p-ERK1/2 and antiERK1/2 were purchased from Cell Signaling Technology (Danvers, USA), and antibody against K-RAS was purchased from Santa Cruz (Santa Cruz, USA). TMZ (Sigma-Aldrich, USA) was used for in vitro chemosensitivity assay.

\section{Real-Time PCR}

RNAs were isolated from human specimens and cells using Trizol (Invitrogen, USA). To measure expression levels of miR-199a (U6 as internal control) and mRNA levels of K-RAS(GAPDH as internal control), the cDNAs were amplified by Real-time PCR with SYBR Green reagents (Vazyme, China) on a 7900HT system(Applied Biosystems), and fold changes were analyzed by relative quantification $\left(2^{-\Delta \Delta \mathrm{Ct}}\right)$.

Primers for K-RAS and GAPDH as below:

K-RAS: Forward Primer: ACAGAGAGTGGAGGATGCTTT, Reverse Primer: TTTCACACAGCCAGGAGTCTT; GAPDH: Forward Primer: ACAACTTTGGTATCGTGGAAGG, Reverse Primer: GCCATCACGCCACAGTTTC.

\section{Immunoblotting}

According to the manufacturer's instruction, cell lysates in this study were prepared using RIPA buffer and indicated protease inhibitors. Aliquots of protein lysates from treated cells were fractionated by SDS-PAGE, after electrophoresis, transferred to a PVDF membrane (Roche, Switzerland), and then subjected to immunoblotting analysis.

\section{Cell Proliferation Assay}

Indicated miR-NC and miR-199a stable cell lines were plated for $2 \times 10^{3}$ cells per well. To evaluate the proliferation activity
A

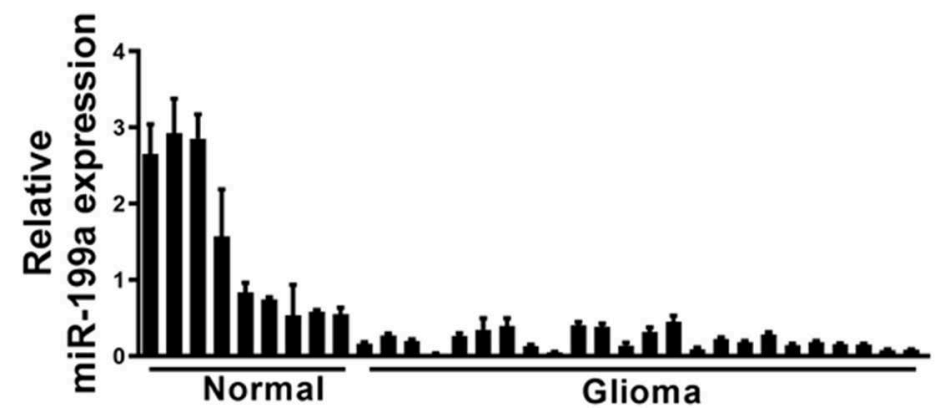

B

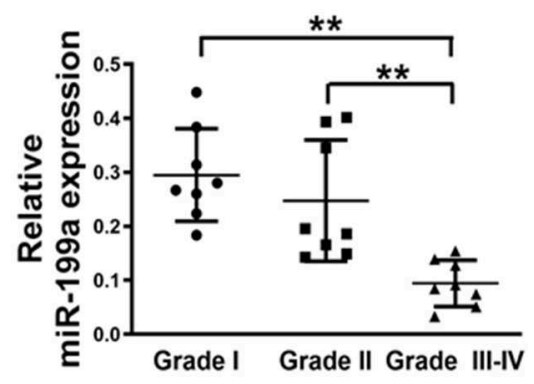

FIGURE 1 | Loss of MiR-199a in human glioma specimens. (A) Relative miR-199a expression levels were analyzed by Real-time RT-PCR in glioma specimens ( $n=$ 24) and adjacent normal tissues ( $n=9)$. U6 RNA levels were used as an internal control; (B) Relative expression levels of miR-199a in cancer tissues at Grades I, II and III-IV (for each grade, $n=8$ ). Data represent mean \pm SD. from three replicates. ${ }^{\star \star}$ Indicates significant difference at $p<0.01$ when compared Grade I or Grade II group with Grade III-IV group. 
of miR-199a in glioma cells, according to the manufacturer's instruction, cell proliferation rate was analyzed with CCK-8 kit (Dojindo Laboratories, Japan).

\section{Migration Assay}

Migration assay was conducted with migration chambers (BD Biosciences, UK). $5 \times 10^{4}$ cells were plated per well in the upper chamber without serum, and the lower chamber was filled with DMEM medium with $10 \%$ FBS. 18-20 h later, the bottom cells were fixed and stained, while non-invading cells were removed. Finally, cells were extracted by $33 \%$ acetic acid and quantitatively detected (OD at $570 \mathrm{~nm})$.

\section{Luciferase Reporter Assay}

3'-UTR region of K-RAS containing software predicted miR199a-matching seed sites (WT) and corresponding mutant sites (mut) were amplified by High fidelity PCR, and inserted into pMIR-REPORTER vector (Ambion, USA). Dual-luciferase activities were analyzed in U87 cells by the Reporter Assay (Promega, USA).

\section{Apoptosis Assay}

Apoptosis assay (BD Pharmingen) in indicated cells were conducted according to the manufacturer's instruction with AnnexinV staining. Then samples were analyzed by flow cytometry (FACS Canto II, BD Biosciences). These data were analyzed by FlowJo software.

\section{In vivo Tumor Growth Assay}

Nude mice (4-weeks-old) were purchased from Animal Center (Shanghai, China), and then bred in special pathogen-free condition. Cells $\left(5 \times 10^{6}\right)$ were then injected subcutaneously into the posterior flanks of each nude mouse. Tumor sizes were measured by vernier caliper using the formula, that is volume
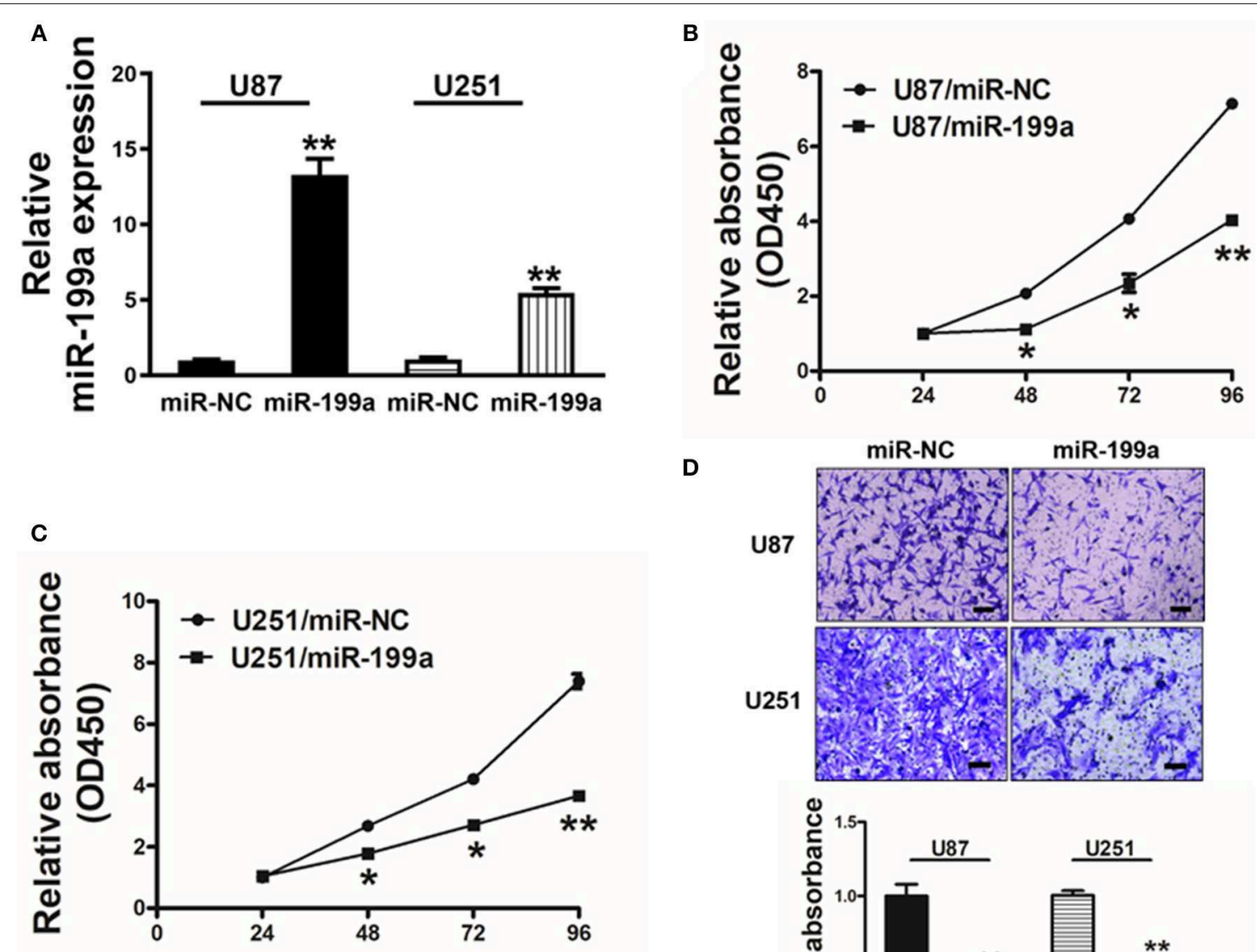

D
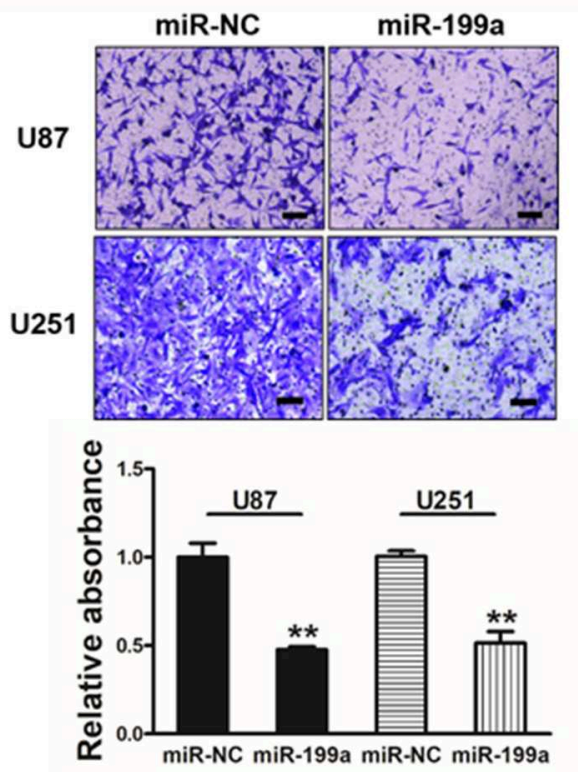

FIGURE 2 | Forced overexpression of miR-199a inhibited cell proliferation and migration in human glioma U87 and U251 cells. (A) Relative expression levels of miR-199a in U87 and U251 stable cell lines were determined by real-time RT-PCR; (B,C) Cells were plated with 2,000 cells per well in 96-well plates, and cell proliferation was determined using Cell Counting Kit-8 (CCK-8) by detecting the absorbance at $450 \mathrm{~nm}$ at indicated time points; (D) Migration assay of cells were performed as previously described. Data represent mean \pm SD. from three replicates. ${ }^{*}$ Indicates significant difference at $p<0.05$ when compared to miR-NC group; ${ }^{\star *}$ Indicates significant difference at $p<0.01$ when compared to miR-NC group. 
$=0.5 \times\left(\right.$ Length $\times$ Width $\left.^{2}\right)$. Twenty-four days later, mice were sacrificed as well as tumors were dissected. All mice used in this study were sacrificed according to the institutional guidelines and regulations.

\section{Statistical Analysis}

All cellular experiments were performed three times. Data were analyzed with GraphPad Prism 5 software. The correlations between miR-199a and K-RAS in human glioma tissues were analyzed by Pearson's test. The differences were considered as statistically significant at $P<0.05$ by $t$-test.

\section{RESULTS}

\section{Loss of MiR-199a in Human Glioma Specimens}

Since mechanism of miR-199a is not fully understood in glioma, qRT-PCR analysis was then performed to determine indicated

A

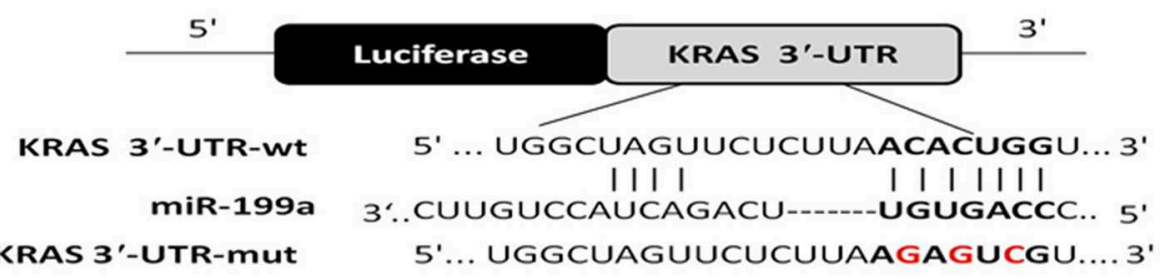

B

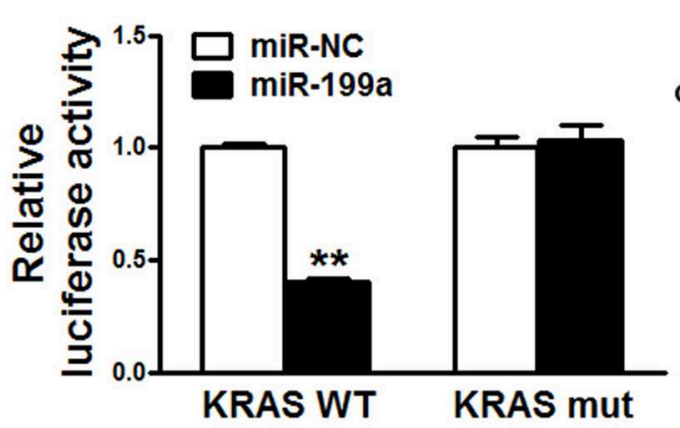

D

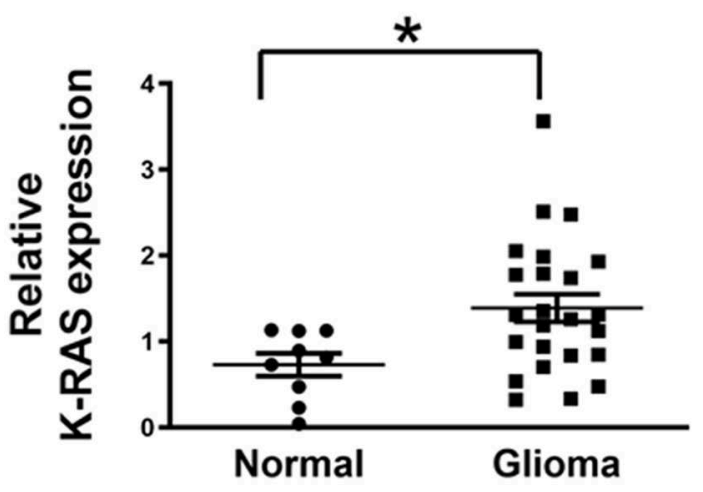

C
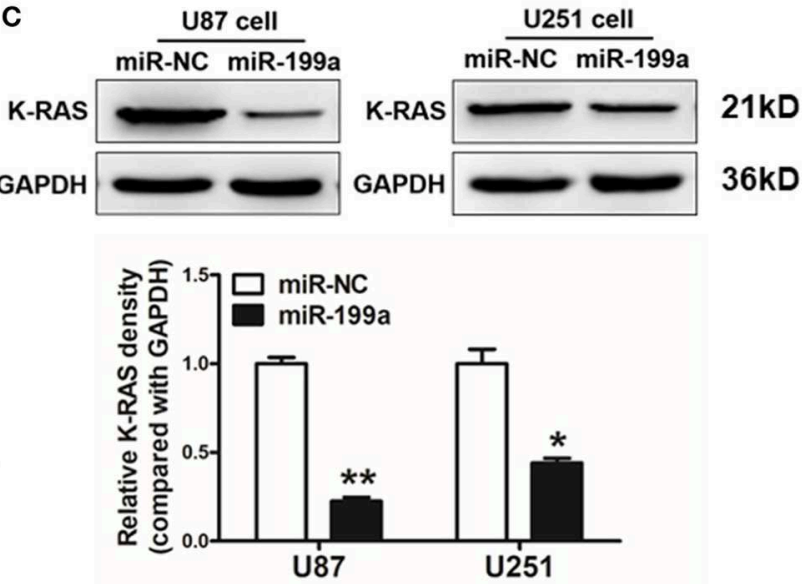

E

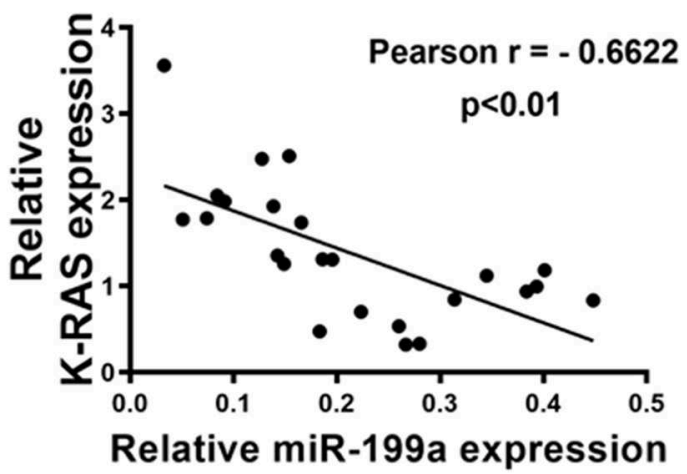

FIGURE 3 | K-RAS is a direct target of miR-199a, and there is a inverse correlation between the expression levels of K-RAS and miR-199a in tumor tissues. (A) The complementary pairings of miR-199a with K-RAS wild-type (WT) and mutant (mut) $3^{\prime}$-UTR reporter constructs are shown. The mutant nucleotides of the K-RAS $3^{\prime}$-UTR region were labeled in red; (B) U87 cells were co-transfected with the reporter constructs containing the WT or mut K-RAS 3'-UTR, miR-NC or miR-199a and pRL-TK plasmids. The luciferase activities were analyzed $24 \mathrm{~h}$ after the transfection; (C) U87 cells were transfected with miR-199a or miR-NC as above. After $72 \mathrm{~h}$, the expression levels of K-RAS and GAPDH in the cells were determined using immunoblotting assay; (D) The expression levels of K-RAS in normal tissues and human glioma specimens were determined by RT-qPCR analysis, and fold changes were obtained by the ratios of K-RAS to GAPDH levels; (E) Pearson's correlation analysis was used to determine the correlation between expression levels of K-RAS and miR-199a in human glioma specimens. These data are means \pm SD. from separate experiments. ${ }^{*}$ ndicates significant difference at $p<0.05$; ${ }^{\star *}$ Indicates significant difference at $p<0.01$. 
expression levels of miR-199a in human glioma specimens. The results clarified that miR-199a expression in tumor $(n=24)$ tissues were significantly lower, as compared to normal $(n=$ 9) tissues (Figure 1A). Furthermore, in tumor tissues of glioma patients, we showed that miR-199a expression were correlated with the clinical stages, which indicated that miR-199a in high grade tumors ( $n=8$, WHO Grades III-IV) were significantly lower when compared to those in low grade tumors $(n=8$, WHO Grade I and $n=8$, WHO Grade II) (Figure 1B). Thus, our results indicated that miR-199a may be a potential novel biomarker for glioma staging.

\section{Forced Overexpression of miR-199a Inhibited Cell Proliferation and Migration Activity in Human Glioma U87 and U251 Cells}

To overexpress miR-199a, human glioma cells U87 and U251 were infected with lentivirus expressing miR-199a, and lentivirus expressing miR-NC was used as control. Stable cell lines which termed as U87/miR-NC, U87/miR199a, U251/miR-NC, and U251/miR-199a were established after puromycin selection, and higher miR-199a expression in U87/miR-199a and U251/miR-199a were demonstrated by qRT-PCR (Figure 2A). Overexpression of miR-199a markedly attenuated cell proliferation activity in U87/miR199a (Figure 2B) and U251/miR-199a cells (Figure 2C). In addition, forced expression of miR-199a markedly reduced cell migration activity (Figure 2D). These results are consistent with the tumor suppressor activities of miR-199a in glioma cells.

\section{K-RAS Is a Direct Target of miR-199a}

TargetScan software was used to predict the direct targets of miR199a, and K-RAS was found to be a potential target (Figure 3A). We constructed luciferase reporter plasmids with either the putative K-RAS wild-type binding sites (WT) or seed sequence mutant sites (mut). Luciferase assay were used to investigate whether the K-RAS is a candidate target of miR-199a. Our results clarified that overexpressing miR-199a in U87 cells reduced the luciferase activity of WT K-RAS reporter by $65 \%$, whereas it did not change the mutant luciferase activities (Figure 3B). Furthermore, forced overexpression of miR-199a significantly attenuated protein expression of K-RAS in U87 and U251 cells (Figure 3C), suggesting that in human glioma cells, miR-199a targets K-RAS directly by binding with its $3^{\prime}$-UTR.

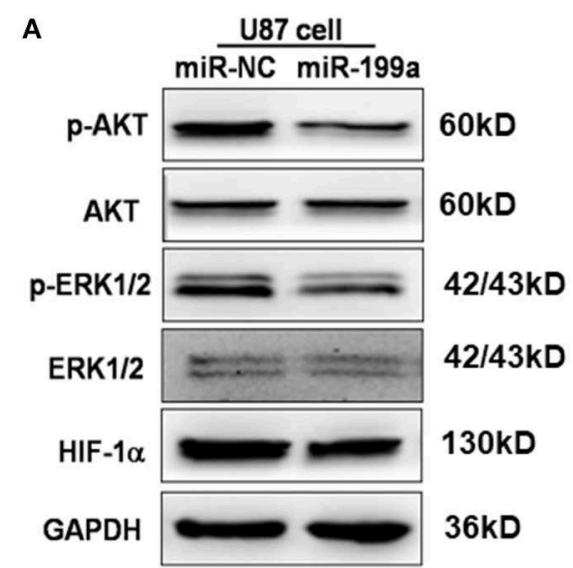

B
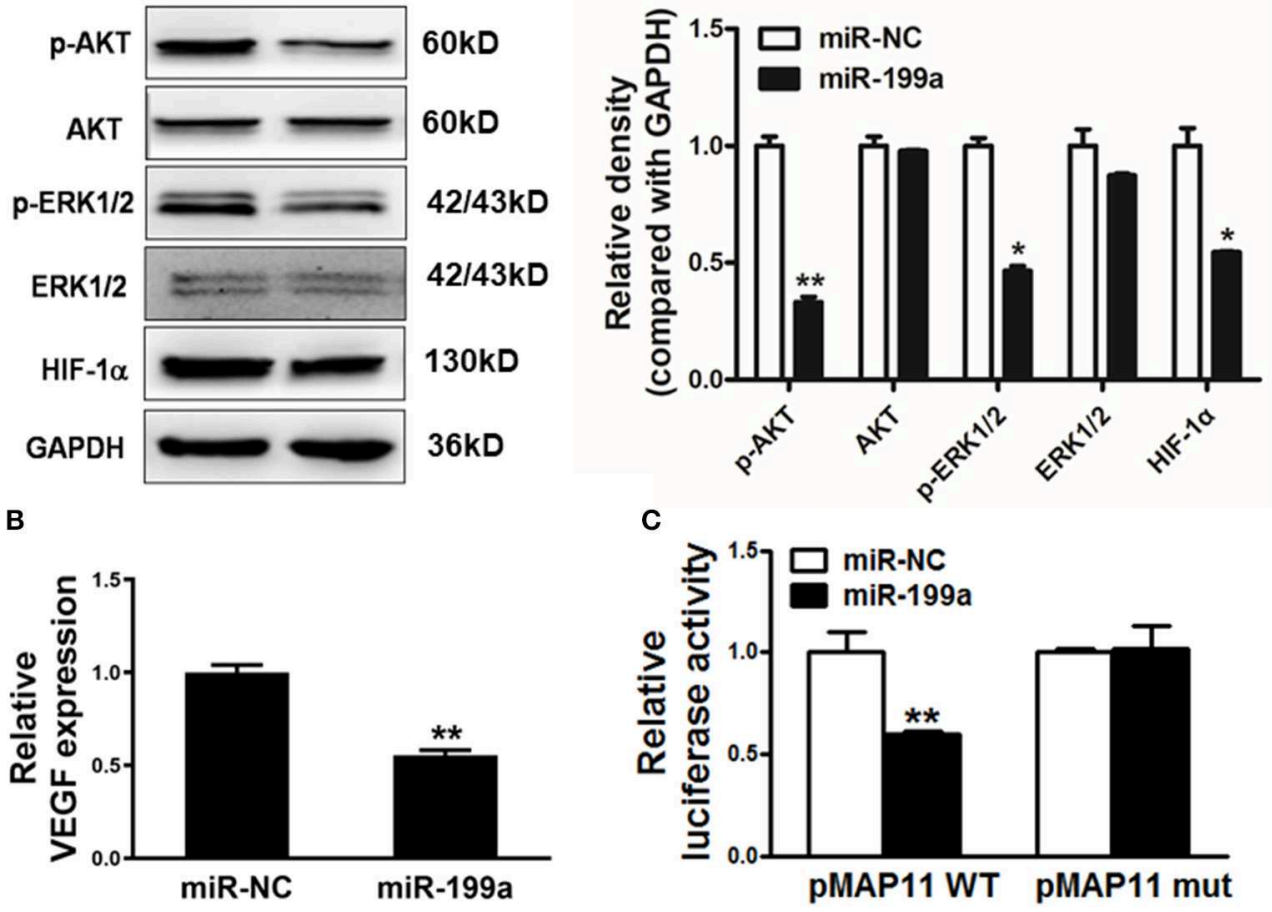

FIGURE 4 | MiR-199a inhibited AKT and ERK1/2 pathways as well as the expression of HIF-1 $\alpha$ and VEGF. (A) The expression levels of phosphorylated AKT (p-AKT), phosphorylated ERK1/2 (p-ERK1/2), total AKT, total ERK1/2 and HIF-1 $\alpha$ in cells were detected by immunoblotting assay; (B) VEGF levels were measured by RT-qPCR in stable cell lines overexpressing miR-NC or miR-199a and normalized to level of GAPDH. *Indicates significant difference at $p<0.05$ when compared to miR-NC group; (C) The wild type VEGF reporter plasmid pMAP11-WT or mutant reporter plasmid pMAP11-mut was co-transfected with pRL-TK plasmid and miR-NC or miR-199a. Firefly and Renilla luciferase activities were measured $24 \mathrm{~h}$ later. Data represent mean $\pm \mathrm{SD}$. of three replicates. ${ }^{\star \star}$ Indicates significant difference at $p<0.01$ when compared to miR-NC group. 


\section{Inverse Correlations of Lower miRNA-199a and Higher K-RAS Levels in Human Glioma Patient Tissues}

To further support the notion that K-RAS oncogene is a direct target, we tested expression levels of K-RAS in human glioma specimens. Our results clarified that K-RAS expression levels were significantly higher in tumor tissues compared with normal tissues (Figure 3D). Furthermore, we determined the correlation between miR-199a and K-RAS oncogene levels in human glioma specimens using Pearson's correlation analysis. Inverse correlation in Figure 3E was found between K-RAS and miR-199a in the human glioma specimens (Pearson's correlation, $r=-0.6622$ ).

\section{Forced Expression of miR-199a Reduced Activation of K-RAS Downstream Molecules AKT and ERK1/2 as Well as HIF-1 $\alpha$ and VEGF Expression Levels in U87 Cells}

The downstream molecules of RAS signaling are AKT and ERK1/2, which are linked to effectors, such as hypoxiainducible factor $1 \alpha$ (HIF-1 $\alpha)$ as well as vascular endothelial growth factor (VEGF). We found that overexpression of miR-199a in U87 cells dramatically suppressed AKT and ERK1/2 activation as indicated by significantly reduced phosphorylated AKT and ERK1/2 levels and a reduction of HIF- $1 \alpha$ protein level without change of total protein levels (Figure 4A).

Accumulated research have shown the importance role of HIF-1 $\alpha /$ VEGF in the regulation of glioma angiogenesis (29, 30). Our previous study has reported that HIF-1 $\alpha$ promotes VEGF gene expression through binding to the hypoxia response element (HRE) in the promoter region of VEGF. In addition to decreased HIF-1 $\alpha$, we also detected a significant reduction of VEGF mRNA levels in miR199a-expressing U87 cells (Figure 4B). To test whether miR-199a inhibited VEGF expression through HIF-1 $\alpha$, we analyzed and compared effects of miR-199a on (1) a VEGF promoter reporter plasmid (pMAP11WT) containing the HIF-1 $\alpha$ binding site, and (2) a mutant (pMAP11 mut) plasmid with mutation, overexpression of miR-199a inhibited the luciferase activities of the VEGF promoter reporter plasmid as shown in Figure 4C. Thus, our results indicated that miR-199a suppressed the gene expression of VEGF through HIF- $1 \alpha$.
A

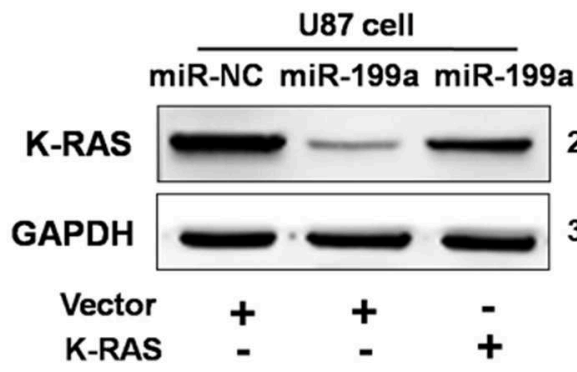

B

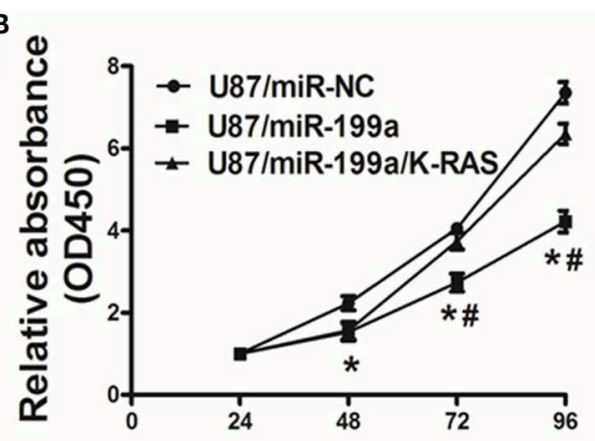

C
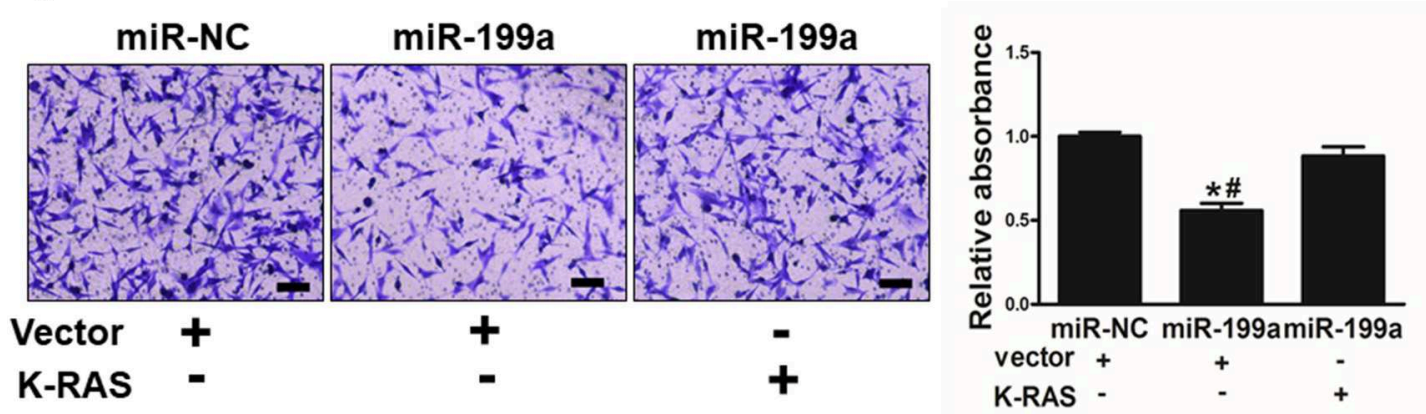

FIGURE 5 | Overexpression of K-RAS cDNA reversed miR-199a-suppressed cell proliferation and cell migration. (A) U87 cells were co-transfected with miR-199a precursor or miR-NC, and empty vector or K-RAS cDNA without 3'-UTR. After $72 \mathrm{~h}$, immunoblotting assay was performed as described above; (B) Cells were treated as above, and cell proliferation assay was determined using CCK-8 kit; (C) U87 cells overexpressing miR-NC or miR-199a were treated as described above, cell migration was determined using 24-well BD migration chambers and quantified using a standard microplate reader (OD at $570 \mathrm{~nm}$ ). Data represent mean \pm SD. of three replicates. ${ }^{*}$ Indicates significant difference at $p<0.05$ compared to miR-NC control; \#Indicates significant difference at $p<0.05$ compared to miR-199a and K-RAS overexpression group. 

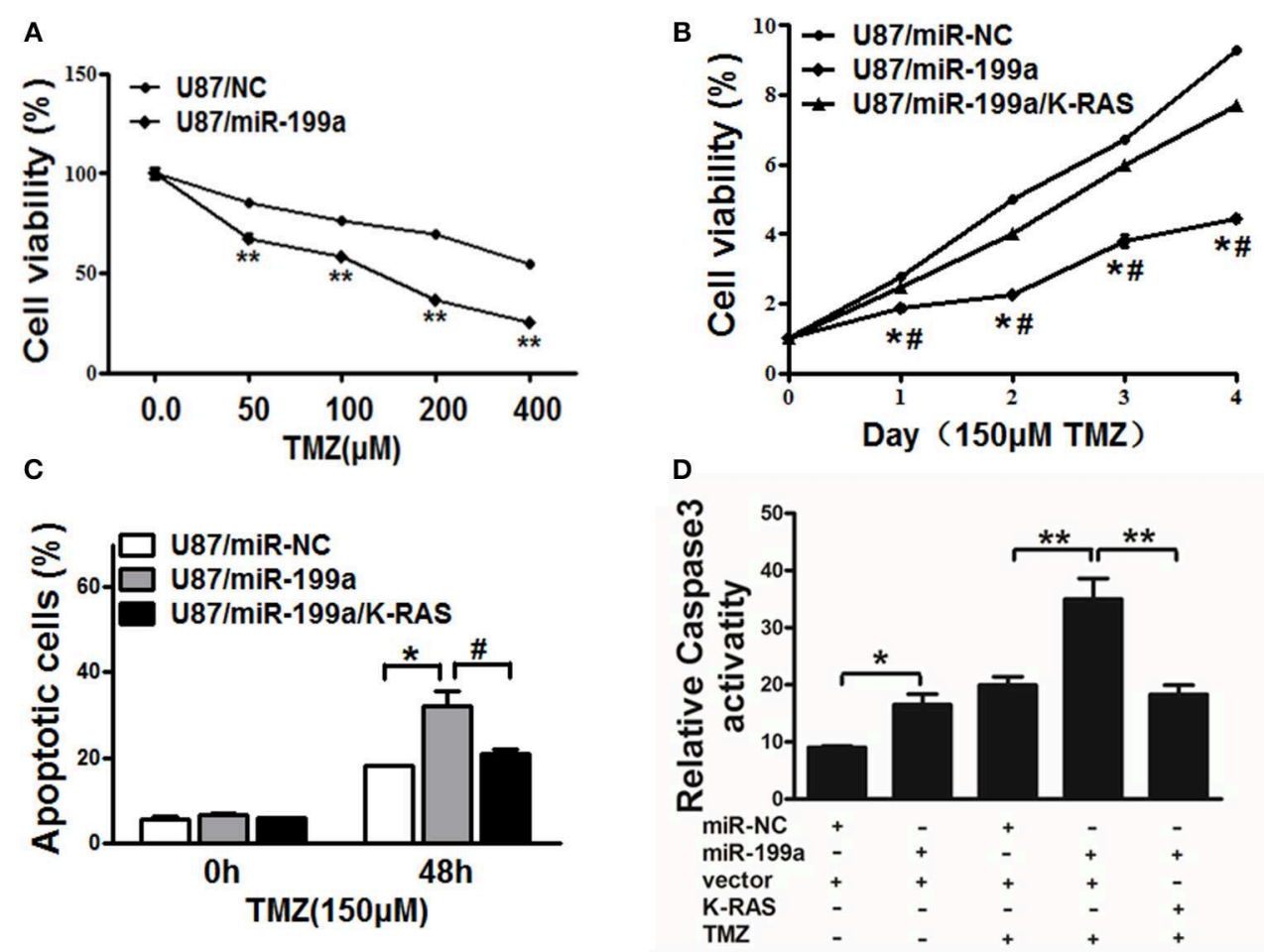

FIGURE 6 | MiR-199a increased TMZ chemosensitivity by targeting K-RAS in glioma cells. (A) U87 cells stably expressing miR-NC or miR-199a were treated with different concentrations of TMZ for 48 h, and cell viability was analyzed using CCK-8 assay; (B) U87 cells stably expressing miR-NC, miR-199a, or miR-199a in combination with K-RAS overexpression were treated with $150 \mu \mathrm{M}$ of TMZ for indicated time points. Cell viability was analyzed by CCK-8 assay; (C,D) Cell apoptosis was analyzed by flow cytometry and by caspase-3 assay. Data represent mean $\pm \mathrm{SD}$. from three replicates. *Indicates significant difference at $p<0.05$ compared to miR-NC control; **Indicates significant difference at $p<0.01$ compared to miR-NC control; \# Indicates significant difference at $p<0.05$ compared to miR-199a and K-RAS overexpression group.

\section{Restoration of K-RAS Reversed miR-199a Mediated Suppression on Cell Proliferation and Migration}

To further demonstrate the role of K-RAS in miR-199a mediated effects on cell proliferation and migration, U87/miR-NC cells or U87/miR-199a cells were co-transfected with control vector (Vector) or K-RAS without 3'-UTR. miR-199a reduced K-RAS expression significantly, and forced expression of K-RAS restored K-RAS expression as shown in Figure 5A. We also determined the effect on cell proliferation activity. As shown in Figure 5B, U87/miR-199a cells has significantly reduced cell proliferation rate, while overexpressing K-RAS in U87/miR-199a cells (U87/miR-199a/K-RAS), restored the cell proliferation rate. The cell migration was determined and quantified by a microplate reader using migration chambers (OD at $570 \mathrm{~nm}$ ). As we expected, overexpression of K-RAS (U87/miR199a/K-RAS) restored miR-199a-inhibited cell migration activity (Figure 5C). To sum up, these results suggested that miR-199a suppresses human glioma cell proliferation and migration, and forced expression of K-RAS reversed miR-199a effects.

\section{Overexpression of miR-199a Rendered Cells More Sensitive to TMZ Through Targeting K-RAS}

TMZ chemoresistance is the major obstacle in process of glioma chemotherapy. In this study, U87/miR-NC cells or U87/miR199a were treated with TMZ at different concentrations for 2 days, as shown in Figure 6A, U87/miR-199a cells were significantly more sensitive to TMZ treatment. Furthermore, overexpressing K-RAS in U87/miR-199a cells (U87/miR-199a/KRAS) nearly completely reversed the chemosensitivity to TMZ treatment (Figure 6B). We further investigated the role of miR199a/K-RAS axis in TMZ mediated apoptosis by apoptosis analysis and caspase-3 activity assay. As shown in Figure 6C, TMZ treatment produced significantly higher apoptotic cell population in U87/miR-199a cells as compare to the control U87/miR-NC cells. Forced expression of K-RAS in U87/miR199a cells (U87/miR-199a/K-RAS) nearly completely reversed the effect. Moreover, activities of caspase- 3 were determined. As shown in Figure 6D, compared to the negative control (Bar 1, $\mathrm{U} 87 / \mathrm{miR}-\mathrm{NC}$ ), overexpression of miR-199a (Bar 2, U87/miR199a) significantly increased caspase 3 activity. TMZ treatmentof 

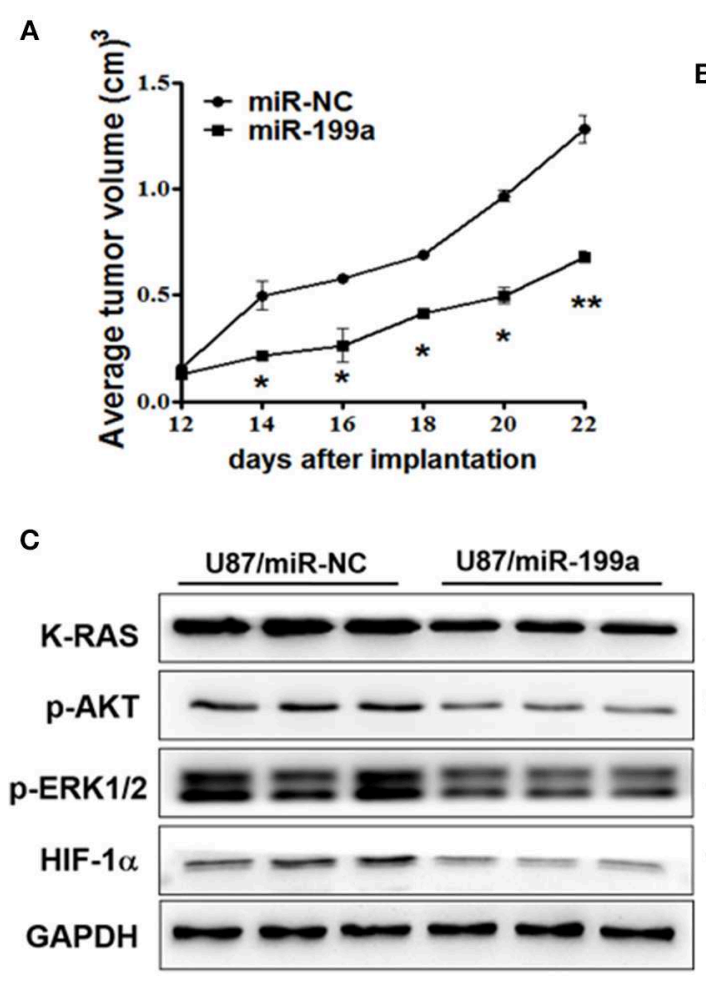

B
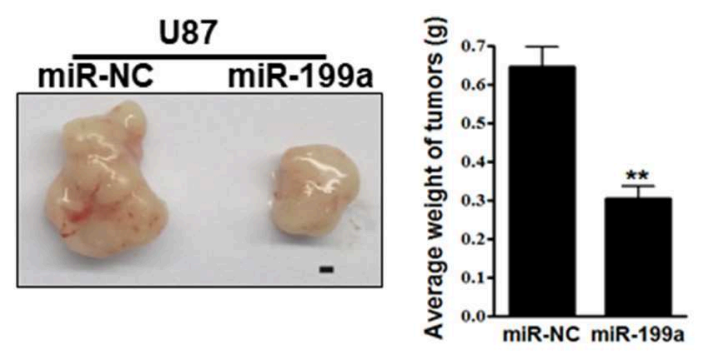

$21 \mathrm{kD}$

$60 \mathrm{kD}$

42/43kD

$130 \mathrm{kD}$

$36 \mathrm{kD}$

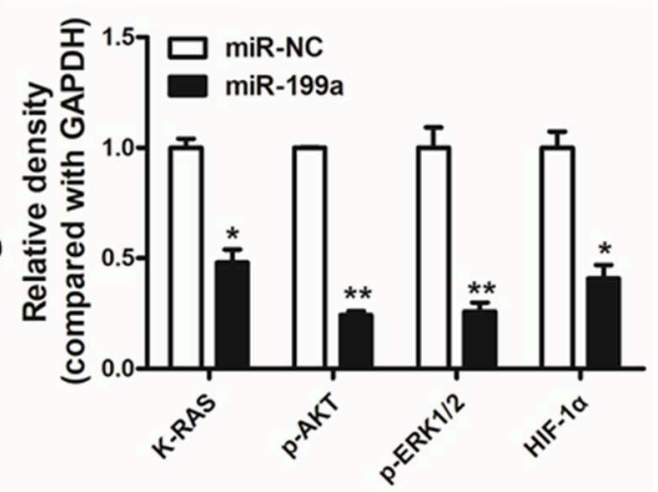

FIGURE 7 | MiR-199a overexpression suppressed tumor growth and decreased expression levels of K-RAS and its downstream molecules in tumor tissues. (A) U87/miR-199a or U87/miR-NC cells (5 $\times 10^{6}$ cells) were dispersed in $100 \mu \mathrm{l}$ of serum-free DMEM medium, and subcutaneously injected into posterior flanks of the nude mice $(n=5)$. Tumors were measured every 2 days after they were apparently detectable on Day 12 . Tumor volumes were calculated using the following formula: volume $=0.5 \times$ (length $\times$ width $^{2}$ ); (B) Mice were sacrificed 24 days after implantation, tumors were harvested and photographed. Representative pictures from each group were shown (Bar = $1 \mathrm{~mm}$ ) and tumors were weighted; (C) Total proteins were extracted from xenografts and subjected to immunoblotting assay to test K-RAS, $p$-AKT, p-ERK1/2, HIF-1 $\alpha$, and GAPDH expression levels. Data were presented mean \pm SD. *Indicates significant difference at $p<0.05$ when compared to miR-NC group; ${ }^{* *}$ Indicates significant difference at $p<0.01$ when compared to miR-NC group.

control U87/miR-NC cells increased caspase3 activity (Bar 3, U87/miR-NC + TMZ), and TMZ treatment of U87/miR-199a cells strongly and significantly increased caspase 3 activity (Bar 4, U87/miR-199a + TMZ). In addition, overexpression of KRAS in U87/miR-199a cells attenuated the activation of caspase-3 (Bar 5, U87/miR-199a/K-RAS + TMZ). To sum up, our results showed that miR-199a rendered glioma cells more sensitive to TMZ through targeting K-RAS.

\section{MiR-199a Suppressed Tumor Growth in vivo}

To determine whether over-expression of miR-199a inhibited tumor growth in vivo, U87/miR-NC cells and U87/miR-199a cells were injected into immunodeficient nude mice. After cell injection, tumor sizes were measured. Compared to control mice injected with U87/miR-NC cells, mice injected with U87/miR199a cells developed significantly smaller tumors from Day 14 (Figure 7A). Twenty-four days later, Mice were sacrificed after implantation, tumors were harvested, photographed, and weighed. A pair of representative tumors trimmed out from U87/miR-199a and U87/miR-NC groups, and the average tumor weight were shown in Figure 7B. Forced expression of miR199a produced tumors with significantly lower tumor weight. We also determined the protein levels of K-RAS, p-AKT, pERK1/2 and HIF- $1 \alpha$ in tumor tissues, and found that these proteins from U87/miR-199a group were significantly lower than that of U87/miR-NC group, which is consistent with in vitro data (Figure 7C). Our results suggested that miR-199a inhibited tumor growth by inhibiting K-RAS expression and its downstream molecules.

\section{DISCUSSION}

MicroRNAs (miRNAs) in diverse human cancers have been frequently indicated to be dysregulated $(31,32)$. The miR-199a have reported to be downregulated in multiple malignancies (3335). We first demonstrated that expression levels of miR-199a was downregulated in clinical glioma samples, and function as a tumor suppressor to increase sensitivity to treatment.

The reported miR-199a targets include GRP78, GSK-3 $\beta$, Discoidin domain receptor 1 (DDR1), mTOR, CD44, and IКB kinase-beta. It works through targeting GRP78, a major 
endoplasmic reticulum chaperone, in prostate cancer cells to induce apoptosis and increase sensitivity to trichostatin $\mathrm{A}$, the histone deacetylase inhibitor (36); through GSK-3 $\beta$ in renal cell cancer cells to decreases cell proliferation (37); through a receptor tyrosine kinase DDR1, to suppress invasiveness and migratory ability of colorectal cancer cells (38); through the mTOR and CD44 to increase sensitivity to cisplatin treatment and to reduce the number of ovarian cancer stem cells $(39,40)$. Finally, it works through targeting ІкB kinase-beta to increase TNF- $\alpha$-induced ovarian cancer cell apoptosis (41). Here, we first identified K-RAS as a novel target of miR-199a. We also confirm the inverse correlation between miR-199a and K-RAS levels in glioma specimens.

Temozolomide (TMZ) is a first-line drug for glioma treatment. A recent study has shown that miR-29c contributed to sensitize cells to temozolomide treatment by targeting $\mathrm{O}^{6}$ methylguanine-DNA methyltransferases in glioma (42). On the other hand, miR-423-5p was reported to function as a oncogene and promoted chemoresistance to temozolomide in glioblastomas (43). Here we found that overexpression of miR199a rendered cells more sensitive to TMZ through its target K-RAS. Thus, miR-199a/K-RAS signaling may be a potential new target to overcome chemoresistance to TMZ in glioma.

\section{CONCLUSIONS}

To sum up, we have clarified that K-RAS is a novel direct target of miR-199a. MiR-199a inhibit activity of cell proliferation, cell migration, drug chemoresistance and tumor growth by

\section{REFERENCES}

1. Chen J, Li Y, Yu TS, McKay RM, Burns DK, Kernie SG, et al. A restricted cell population propagates glioblastoma growth after chemotherapy. Nature. (2012) 488:522-6. doi: 10.1038/nature11287

2. Del Vecchio CA, Li G, Wong AJ. Targeting EGF receptor variant III: tumorspecific peptide vaccination for malignant gliomas. Expert Rev Vaccines. (2012) 11:133-44. doi: 10.1586/erv.11.177

3. Fuller GN, Scheithauer BW. The 2007 Revised World Health Organization (WHO) classification of tumours of the central nervous system: newly codified entities. Brain Pathol. (2007) 17:304-7. doi: 10.1111/j.1750-3639.2007.00084.x

4. Louis DN, Ohgaki H, Wiestler OD, Cavenee WK, Burger PC, Jouvet A, et al. The 2007 WHO classification of tumours of the central nervous system. Acta Neuropathol. (2007) 114:97-109. doi: 10.1007/s00401-007-0243-4

5. Zhang L, Wu X, Xu T, Luo C, Qian J, Lu Y. Chemotherapy plus radiotherapy versus radiotherapy alone in patients with anaplastic glioma: a systematic review and meta-analysis. J Cancer Res Clin Oncol. (2013) 139:719-26. doi: 10.1007/s00432-013-1387-3

6. Van Meir EG, Hadjipanayis CG, Norden AD, Shu HK, Wen PY, Olson JJ. Exciting new advances in neuro-oncology: the avenue to a cure for malignant glioma. CA Cancer J Clin. (2010) 60:166-93. doi: 10.3322/caac.20069

7. Wen PY, Kesari S. Malignant gliomas in adults. $N$ Engl J Med. (2008) 359:492-507. doi: 10.1056/NEJMra 0708126

8. Jiang CF, Shi ZM, Li DM, Qian YC, Ren Y, Bai XM, et al. Estrogen-induced miR-196a elevation promotes tumor growth and metastasis via targeting SPRED1 in breast cancer. Mol Cancer. (2018) 17:83. doi: 10.1186/s12943-018-0830-0

9. Niu XB, Fu GB, Wang L, Ge X, Liu WT, Wen YY, et al. Insulinlike growth factor-I induces chemoresistence to docetaxel by regulating K-RAS via AKT and ERK signalings. These results elucidated that miR-199a/K-RAS in the future may be used as a target for glioma treatment.

\section{DATA AVAILABILITY STATEMENT}

The datasets generated for this study are available on request to the corresponding author.

\section{ETHICS STATEMENT}

The studies involving human participants were reviewed and approved by Ethics Committee of Nanjing University. The patients/participants provided their written informed consent to participate in this study. The animal study was reviewed and approved by Ethics Committee of Nanjing University.

\section{AUTHOR CONTRIBUTIONS}

WL, LW, and XG carried out the samples collection and performed the experiments. $\mathrm{X}-\mathrm{BJ}$ and $\mathrm{L}-\mathrm{HW}$ revised the manuscript. W-TL, LC, ZZ, Z-MS, L-ZL, and ML designed the studies. WL, LW, J-YC, and B-HJ wrote the manuscript.

\section{FUNDING}

This work was supported in part by National Natural Science Foundation of China (81502170, 81772951, 81803197). inhibiting miR-143 in human prostate cancer. Oncotarget. (2017) 8:107157-66. doi: 10.18632/oncotarget.22362

10. Ambros V, Lee RC. Identification of microRNAs and other tiny noncoding RNAs by cDNA cloning. Methods Mol Biol. (2004) 265:131-58. doi: 10.1385/1-59259-775-0:131

11. Pillai RS, Bhattacharyya SN, Filipowicz W. Repression of protein synthesis by miRNAs: how many mechanisms? Trends Cell Biol. (2007) 17:118-26. doi: 10.1016/j.tcb.2006.12.007

12. Thai TH, Christiansen PA, Tsokos GC. Is there a link between dysregulated miRNA expression and disease? Discov Med. (2010) 10:184-94.

13. German MA, Pillay M, Jeong DH, Hetawal A, Luo S, Janardhanan P, et al. Global identification of microRNA-target RNA pairs by parallel analysis of RNA ends. Nat Biotechnol. (2008) 26:941-6. doi: 10.1038/nbt1417

14. Bartel DP. MicroRNAs: genomics, biogenesis, mechanism, and function. Cell. (2004) 116:281-97. doi: 10.1016/s0092-8674(04)00045-5

15. Gibson NW. Engineered microRNA therapeutics. J R Coll Physicians Edinb. (2014) 44:196-200. doi: 10.4997/JRCPE.2014.302

16. Wang L, Shi ZM, Jiang CF, Liu X, Chen QD, Qian X, et al. MiR143 acts as a tumor suppressor by targeting N-RAS and enhances temozolomide-induced apoptosis in glioma. Oncotarget. (2014) 5:5416-27. doi: 10.18632/oncotarget.2116

17. Shi Z, Chen Q, Li C, Wang L, Qian X, Jiang C, et al. MiR-124 governs glioma growth and angiogenesis and enhances chemosensitivity by targeting R-Ras and N-Ras. Neuro Oncol. (2014) 16:1341-53. doi: 10.1093/neuonc/nou084

18. Byrnes KA, Phatak P, Mansour D, Xiao L, Zou T, Rao JN, et al. Overexpression of miR-199a-5p decreases esophageal cancer cell proliferation through repression of mitogen-activated protein kinase kinase kinase-11 (MAP3K11). Oncotarget. (2016) 7:8756-70. doi: 10.18632/oncotarget.6752

19. Guo W, Qiu Z, Wang Z, Wang Q, Tan N, Chen T, et al. MiR-199a-5p is negatively associated with malignancies and regulates glycolysis and lactate 
production by targeting hexokinase 2 in liver cancer. Hepatology. (2015) 62:1132-44. doi: 10.1002/hep.27929

20. Kim BK, Yoo HI, Kim I, Park J, Kim Yoon S. FZD6 expression is negatively regulated by miR-199a-5p in human colorectal cancer. BMB Rep. (2015) 48:360-6. doi: 10.5483/bmbrep.2015.48.6.031

21. Lee JM, Heo MJ, Lee CG, Yang YM, Kim SG. Increase of miR199a-5p by protoporphyrin IX, a photocatalyzer, directly inhibits E2F3, sensitizing mesenchymal tumor cells to anti-cancer agents. Oncotarget. (2015) 6:3918-31. doi: 10.18632/oncotarget.2928

22. Mussnich P, Rosa R, Bianco R, Fusco A, D’Angelo D. MiR199a-5p and miR-375 affect colon cancer cell sensitivity to cetuximab by targeting PHLPP1. Expert Opin Ther Targets. (2015) 19:1017-26. doi: 10.1517/14728222.2015.1057569

23. Song J, Gao L, Yang G, Tang S, Xie H, Wang Y, et al. MiR-199a regulates cell proliferation and survival by targeting FZD7. PLoS ONE. (2014) 9:e110074. doi: 10.1371/journal.pone.0110074

24. Hall A. The cellular functions of small GTP-binding proteins. Science. (1990) 249:635-40.

25. Milinkovic VP, Skender Gazibara MK, Manojlovic Gacic EM, Gazibara TM, Tanic NT. The impact of TP53 and RAS mutations on cerebellar glioblastomas. Exp Mol Pathol. (2014) 97:202-7. doi: 10.1016/j.yexmp.2014.07.009

26. Ryu MJ, Liu Y, Zhong $\mathrm{X}$, Du J, Peterson $\mathrm{N}$, Kong G, et al. Oncogenic Kras expression in postmitotic neurons leads to S100A8S100A9 protein overexpression and gliosis. J Biol Chem. (2012) 287:22948-58. doi: 10.1074/jbc.M112.357772

27. Bodemann BO, White MA. Ral GTPases and cancer: linchpin support of the tumorigenic platform. Nat Rev Cancer. (2008) 8:133-40. doi: 10.1038/nrc2296

28. Schubbert S, Shannon K, Bollag G. Hyperactive Ras in developmental disorders and cancer. Nat Rev Cancer. (2007) 7:295-308. doi: 10.1038/nr c2109

29. Gillespie DL, Whang K, Ragel BT, Flynn JR, Kelly DA, Jensen RL. Silencing of hypoxia inducible factor-1alpha by RNA interference attenuates human glioma cell growth in vivo. Clin Cancer Res. (2007) 13:24418. doi: 10.1158/1078-0432.CCR-06-2692

30. Zagzag D, Lukyanov Y, Lan L, Ali MA, Esencay M, Mendez O, et al. Hypoxia-inducible factor 1 and VEGF upregulate CXCR4 in glioblastoma: implications for angiogenesis and glioma cell invasion. Lab Invest. (2006) 86:1221-32. doi: 10.1038/labinvest.3700482

31. Liu LZ, Li C, Chen Q, Jing Y, Carpenter R, Jiang Y, et al. MiR21 induced angiogenesis through AKT and ERK activation and HIF1alpha expression. PLoS ONE. (2011) 6:e19139. doi: 10.1371/journal.pone.00 19139

32. Xu Q, Liu LZ, Qian X, Chen Q, Jiang Y, Li D, et al. MiR-145 directly targets p70S6K1 in cancer cells to inhibit tumor growth and angiogenesis. Nucleic Acids Res. (2012) 40:761-74. doi: 10.1093/nar/gkr730

33. He XJ, Ma YY, Yu S, Jiang XT, Lu YD, Tao L, et al. Up-regulated miR-199a-5p in gastric cancer functions as an oncogene and targets klotho. BMC Cancer. (2014) 14:218. doi: 10.1186/1471-2407-14-218
34. Song G, Zeng H, Li J, Xiao L, He Y, Tang Y, et al. miR-199a regulates the tumor suppressor mitogen-activated protein kinase kinase kinase 11 in gastric cancer. Biol Pharm Bull. (2010) 33:1822-7. doi: 10.1248/bpb.33.1822

35. Zhang Y, Fan KJ, Sun Q, Chen AZ, Shen WL, Zhao ZH, et al. Functional screening for miRNAs targeting Smad4 identified miR-199a as a negative regulator of TGF-beta signalling pathway. Nucleic Acids Res. (2012) 40:9286-97. doi: 10.1093/nar/gks667

36. Su SF, Chang YW, Andreu-Vieyra C, Fang JY, Yang Z, Han B, et al. miR30d, miR-181a and miR-199a-5p cooperatively suppress the endoplasmic reticulum chaperone and signaling regulator GRP78 in cancer. Oncogene. (2013) 32:4694-701. doi: 10.1038/onc.2012.483

37. Tsukigi M, Bilim V, Yuuki K, Ugolkov A, Naito S, Nagaoka A, et al. Re-expression of miR-199a suppresses renal cancer cell proliferation and survival by targeting GSK-3beta. Cancer Lett. (2012) 315:189-97. doi: 10.1016/j.canlet.2011.10.008

38. Hu Y, Liu J, Jiang B, Chen J, Fu Z, Bai F, et al. MiR-199a-5p loss up-regulated DDR1 aggravated colorectal cancer by activating epithelialto-mesenchymal transition related signaling. Dig Dis Sci. (2014) 59:2163-72. doi: 10.1007/s10620-014-3136-0

39. Cheng W, Liu T, Wan X, Gao Y, Wang H. MicroRNA-199a targets CD44 to suppress the tumorigenicity and multidrug resistance of ovarian cancer-initiating cells. FEBS J. (2012) 279:2047-59. doi: 10.1111/j.1742-4658.2012.08589.x

40. Wang Z, Ting Z, Li Y, Chen G, Lu Y, Hao X. microRNA-199a is able to reverse cisplatin resistance in human ovarian cancer cells through the inhibition of mammalian target of rapamycin. Oncol Lett. (2013) 6:789-94. doi: 10.3892/ol.2013.1448

41. Chen R, Alvero AB, Silasi DA, Kelly MG, Fest S, Visintin I, et al. Regulation of IKKbeta by miR-199a affects NF-kappaB activity in ovarian cancer cells. Oncogene. (2008) 27:4712-23. doi: 10.1038/onc.2008.112

42. Xiao S, Yang Z, Qiu X, Lv R, Liu J, Wu M, et al. miR-29c contribute to glioma cells temozolomide sensitivity by targeting O6methylguanine-DNA methyltransferases indirectely. Oncotarget. (2016) 7:50229-38. doi: 10.18632/oncotarget.10357

43. Li S, Zeng A, Hu Q, Yan W, Liu Y, You Y. miR-423-5p contributes to a malignant phenotype and temozolomide chemoresistance in glioblastomas. Neuro Oncol. (2016) 19:55-65. doi: 10.1093/neuonc/now129

Conflict of Interest: The authors declare that the research was conducted in the absence of any commercial or financial relationships that could be construed as a potential conflict of interest.

Copyright (c) 2019 Li, Wang, Ji, Wang, Ge, Liu, Chen, Zheng, Shi, Liu, Lin, Chen and Jiang. This is an open-access article distributed under the terms of the Creative Commons Attribution License (CC BY). The use, distribution or reproduction in other forums is permitted, provided the original author(s) and the copyright owner(s) are credited and that the original publication in this journal is cited, in accordance with accepted academic practice. No use, distribution or reproduction is permitted which does not comply with these terms. 